Utilisation des ciments orthopédiques dans un centre hospitalier universitaire multisite

\title{
Bone Cement Usage Modalities at a Multi-Site University Hospital Centre
}

Marine BARRAL ${ }^{\mathrm{a}}$, Laure LALANDE ${ }^{\mathrm{a}}$, Anthony VISTE ${ }^{\mathrm{b}, \mathrm{c}}$, Jean-Luc BESSE ${ }^{\mathrm{b}, \mathrm{c}}$, Michel-Henri FESSY $^{\mathrm{b}, \mathrm{c}}$, Emmanuelle CARRE $^{\mathrm{a}, *}$

a Hospices Civils de Lyon, Centre Hospitalier Lyon-Sud, Service de Pharmacie, 165 chemin du Grand Revoyet, 69495 Pierre-Bénite Cedex, France.

b Hospices Civils de Lyon, Centre Hospitalier Lyon-Sud, Service de Chirurgie Orthopédique et Traumatologique, 165 chemin du Grand Revoyet, 69495 Pierre-Bénite Cedex, France.

c Université Lyon 1, IFSTTAR, LBMC UMR-T 9406 - Laboratoire de Biomécanique et Mécanique des Chocs, 69675 Bron Cedex, France

* Auteur correspondant : Emmanuelle Carré - Hospices Civils de Lyon, Centre Hospitalier Lyon-Sud, Service de Pharmacie, 165 chemin du Grand Revoyet, 69495 Pierre-Bénite Cedex, France.

emmanuelle.carre@chu-lyon.fr

Tél : +33 (0)4 78865123

Fax : +33(0)4 78861991

Ne pas utiliser, pour citation, la référence française de cet article, mais celle de l'article original paru dans Orthopaedics \&Traumatology: Surgery \& Research, en utilisant le DOI ci-dessus. 


\section{Résumé}

Introduction :

Les ciments sont régulièrement utilisés en chirurgie orthopédique lors des arthroplasties mais peu de recommandations existent concernant leur bon usage, alors que celui-ci conditionne les résultats à long terme de la chirurgie. Les modalités d'utilisation des ciments dans des centres hospitaliers français ne sont pas connues avec précision. Nous avons donc mené une enquête prospective au sein d'un centre hospitalier universitaire (CHU) multisite afin de : 1) connaître les pratiques, 2) distinguer si des différences sont observées selon l'expérience des chirurgiens, 3) préciser d'éventuelles différences par rapport aux recommandations.

Hypothèse :

Il existe une grande hétérogénéité des pratiques de cimentation dans un centre universitaire.

Matériel et méthodes :

Une enquête, à l'aide d'un questionnaire, a été diffusée auprès de 5 services de chirurgie orthopédique de notre établissement. D'une part, les chirurgiens renseignaient âge, sexe, nombre d'années d'expérience, type d'implants, nombre de poses annuelles par type d'implant et par indication, et taux de prothèses cimentées. D'autre part, ils précisaient la référence de ciment utilisée et leur technique de cimentation.

\section{Résultats :}

Vingt-et-un chirurgiens orthopédiques ont répondu à l'enquête. Vingt sur 21 étaient des hommes, d'une moyenne d'âge de 41 ans [31-59] et 11 ans [1-29] d'expérience. Les chirurgiens utilisaient majoritairement (20/21 (95\%)) un ciment de haute viscosité imprégné d'antibiotique, surtout dans l'arthroplastie du genou avec un nombre médian de 55 [0-218] poses primaires par an et 8 [1-40] reprises. Les techniques de cimentation en air ambiant étaient variées : $57 \%$ (12/21) utilisaient le lavage pulsé pour préparer l'os avant cimentation, $86 \%$ (18/21) réalisaient une double cimentation (os et implant). Parmi les 18 chirurgiens qui pratiquaien l'arthroplastie du genou, 9/11 des chirurgiens expérimentés (> 5 ans d'expérience) utilisaient plus le lavage pulsé contre seulement 3/7 des chirurgiens moins expérimentés. De même, 11/12 des utilisateurs habituels du ciment (> 15 prothèses cimentées par an) utilisaient plus le lavage pulsé contre seulement 1/6 des utilisateurs occasionnels du ciment. 


\section{Discussion :}

Les techniques de cimentation étaient hétérogènes, du fait du peu de recommandations et d'une littérature controversée. Pour notre établissement, une amélioration de la préparation de l'os avec utilisation du lavage pulsé et de la double cimentation sont à encourager puisque ces pratiques font consensus dans la littérature. Pour les autres étapes de cimentation, notre étude montre la nécessité d'un partage d'expérience pour harmoniser les pratiques et promouvoir une utilisation optimale des ciments.

Level of evidence: IV, enquête par questionnaire.

Mots-clés : ciment orthopédique ; enquête ; bon usage ; technique de cimentation 


\section{Introduction}

Les ciments acryliques, utilisés en chirurgie orthopédique sont des polymères pour lesquels, malgré leur utilisation ancienne, il n'existe pas aujourd'hui de technique de cimentation de référence puisque différentes méthodes de préparation et d'application du ciment sont décrites dans la littérature [1,2]. Or la technique de cimentation et le choix du type de ciment sont identifiés parmi les causes de descellement aseptique [2], une des principales causes de révision dans l'arthroplastie de genou ou de hanche (29-45\%) [3-5]. Ainsi, leur bonne utilisation est un élément de réussite de l'arthroplastie à long terme [6].

En France, la formation des chirurgiens orthopédistes à l'utilisation des ciments est peu développée et très peu de recommandations existent sur les techniques de cimentation. L'arrivée de prothèses sans ciment sur le marché, notamment dans l'arthroplastie de la hanche, n'encourage pas l'amélioration de leur formation et peut expliquer le manque d'expérience des chirurgiens autour de la préparation du ciment, la préparation de l'os ou encore la technique d'application. Quelques enquêtes de pratiques anciennes sur la technique de cimentation ont été menées à l'étranger [7-9] mais les pratiques dans les centres hospitaliers français ne sont pas connues avec précision.

Aussi, nous avons mené une enquête prospective auprès des chirurgiens orthopédistes d'un centre hospitalier universitaire (CHU) multisite afin de : 1) connaître les pratiques, 2) distinguer si des différences sont observées selon l'expérience des chirurgiens, 3). préciser d'éventuelles différences par rapport aux recommandations. Nous supposons qu'il existe une grande hétérogénéité des pratiques de cimentation dans un CHU français.

\section{Matériel et méthodes}

\subsection{Matériel}

Une enquête descriptive a été menée auprès des chirurgiens orthopédistes de notre établissement. Après analyse des données de la littérature et des recommandations de bonnes pratiques de cimentation, un questionnaire a été élaboré par un interne en pharmacie (MB) et un pharmacien praticien hospitalier (EC), en charge des dispositifs médicaux implantables depuis 2003, puis validé par un chirurgien orthopédiste sénior (AV) (Appendix). La première partie du questionnaire concernait le chirurgien lui-même, âge, sexe, nombre d'années 
d'expérience, type d'implants (prothèse de hanche, de genou, d'épaule, de cheville, autre), nombre de poses annuelles par type d'implant et par indication(s) (fixation implant primaire, reprise, utilisation comme espaceur), taux de prothèses cimentées. La seconde partie portait sur la référence du (des) ciment(s) utilisé(s) et la technique de cimentation employée (préparation, application, pressurisation).

\subsection{Méthodes}

Le questionnaire a été diffusé par email aux chefs de service des cinq unités de chirurgie orthopédique de notre hôpital multisite, 1 service de chirurgie orthopédique pédiatrique et 4 services de chirurgie orthopédique adulte. Il leur était demandé de transmettre ce questionnaire à tous les chirurgiens de leur équipe et de le retourner, complété. La diffusion a eu lieu le 31 janvier 2017, une relance a été faite un mois plus tard, et la date limite de retour à la pharmacie était fixée au 31 mars 2017. Un complément d'enquête en 2018 a permis d'affiner l'activité chirurgicale réelle sur 2017. En cas de données manquantes, nous avons eu recours aux données de traçabilité des implants.

\subsection{Analyse Statistique}

Les réponses au questionnaire ont été anonymisées puis saisies et analysées avec Excel 2010 ${ }^{\mathrm{TM}}$ (Microsoft, Redmond, Wa, USA). Une analyse statistique descriptive a été réalisée.

\section{Résultats}

Au total, 21 chirurgiens ont répondu à l'enquête (Figure 1). La totalité des répondeurs utilisaient des ciments orthopédiques dans leur pratique courante. Le Tableau 1 résume les principales caractéristiques des répondeurs. Vingt sur 21 répondeurs étaient des hommes, d'une moyenne d'âge de 41 ans [31-59] et 11 ans [1-29] d'expérience en chirurgie orthopédique. Le volume d'activité annuelle par type d'implant et le taux de poses cimentées sont présentés dans le Tableau 2. Les ciments étaient majoritairement utilisés dans l'arthroplastie du genou avec un nombre médian de 55 [0-218] poses primaires par an et 8 [140] reprises. Les techniques de cimentation des différents répondeurs sont présentées dans le Tableau 3. Dans notre hôpital, le ciment majoritairement utilisé (20/21, 95\%) était un ciment de haute viscosité avec gentamicine, sauf pour la chirurgie du membre supérieur où un ciment sans antibiotique était employé. Le ciment était principalement utilisé pour la fixation 
d'implants (primaire ou reprise) par tous les chirurgiens répondeurs (21/21) et occasionnellement comme espaceur (révision prothétique en deux temps) pour 10/21 des répondeurs, conformément aux indications du marquage CE des ciments. Le mélange des ciments était uniquement manuel en air ambiant puisqu'aucun système sous vide n'était référencé. Pour la préparation, le lavage pulsé était utilisé par 12/21 chirurgiens (57\%). L'application du ciment était réalisée au pistolet pour 15 chirurgiens, mais uniquement pour les reprises de prothèses totales de hanche pour 9 d'entre eux, pour les 6 autres, en cas de cimentation diaphysaire des quilles de prothèses totales du genou. La majorité des répondeurs (18/21, 86\%) réalisait une double cimentation, à la fois sur l'os et sur l'implant, dont les 12 chirurgiens qui utilisaient le lavage pulsé. La pressurisation était manuelle, à l'aide d'une compresse, d'une spatule ou avec un pistolet au niveau du fémur (prothèse de hanche). Enfin, pour les prothèses totales de genou, 10 chirurgiens ne cimentaient qu'une partie de la quille tibiale et 8 la totalité. Pour la prothèse de cheville, le ciment n'était pas utilisé pour la pose (implants non cimentés exclusifs en Europe) mais pour le curetage-cimentage des lésions géodiques d'ostéolyse et les espaceurs après dépose de prothèse.

Concernant la hanche et le genou, aucune différence n'a été observée selon le nombre d'années d'expérience des chirurgiens sauf pour l'utilisation du lavage pulsé. En effet, parmi les 18 chirurgiens réalisant des arthroplasties de genou, 4/7 chirurgiens ayant moins d'expérience ( $\leq 5$ ans) n'utilisaient pas le lavage pulsé contre 2/11 des chirurgiens plus expérimentés. Aussi, 5/6 des chirurgiens peu utilisateurs ( $\leq 15$ prothèses cimentées par an) n'utilisaient pas le lavage pulsé contre $1 / 12$ pour les chirurgiens plus utilisateurs.

\section{Discussion}

Cette enquête, la première en France concernant les ciments orthopédiques, nous a permis de mieux définir les pratiques des différentes équipes de chirurgie orthopédique d'un établissement universitaire. Cette étude a confirmé l'hétérogénéité des pratiques, le besoin en formation des chirurgiens orthopédiques au bon usage des ciments et la nécessité d'un partage d'expérience pour une harmonisation des pratiques. Cette formation est d'autant plus nécessaire que les recommandations de bon usage des ciments orthopédiques sont peu nombreuses et les données de la littérature controversées. 
Le ciment majoritairement utilisé dans notre établissement était un ciment à la gentamicine, à haute viscosité, ciment fréquemment utilisé par d'autres équipes étrangères [7-9], ce qui est conforme aux recommandations de la Haute Autorité de Santé [10]. La préparation du ciment orthopédique (mélange liquide - poudre) est importante puisque de celle-ci dépend la qualité finale du ciment, et donc la bonne fixation de l'implant à l'os. Dans notre établissement aucun système de mélange sous vide n'est disponible. De manière générale en France, leur utilisation est peu fréquente contrairement à d'autres pays (Tableau 4). Les principaux freins sont leur coût, variant de 30 à 50 euros, et des données contradictoires dans la littérature sur les bénéfices cliniques [11-13].

La préparation osseuse après coupe et avant cimentation comporte un nettoyage et un séchage de l'os receveur. Le nettoyage abondant de l'os par un système de lavage pulsé est recommandé aussi bien dans l'arthroplastie de hanche que de genou [14-16], il était largement utilisé dans les enquêtes étrangères (Tableau 4). Dans notre établissement, l'enquête a permis de montrer que la technique de lavage pulsé était insuffisamment utilisée, et devrait donc être encouragée.

Les méthodes d'application et de pressurisation des ciments sont variées aussi bien dans notre établissement que dans d'autres équipes chirurgicales (Tableau 4). Tout d'abord, l'application peut être manuelle, à l'aide d'une compresse, du doigt («finger packing »), d'une spatule ou d'une seringue, ou pressurisée à l'aide d'un pistolet [17]. Les données de la littérature sont insuffisantes pour valider la technique de choix : une application manuelle ne permettrait pas une pénétration suffisante alors que les pistolets sembleraient exposer à un risque théorique de dommage thermique et de perte de substance osseuse plus importante en cas de révision prothétique [18-20]. De plus, l'application du ciment peut se faire sur l'implant ou l'os seul, ou en double cimentation (os et implant). Ces différentes techniques ont été comparées, principalement dans l'arthroplastie de genou. La double cimentation semble permettre la meilleure pénétration du ciment dans l'os [2,18]. Enfin, l'étape de pressurisation est indispensable après application du ciment puisqu'elle permet la phase de durcissement final et évite une chute de la pression intra-médullaire. Elle permet également une meilleure pénétration du ciment dans l'os spongieux et réduit la porosité du ciment [21,22]. En l'absence de recommandation claire, une double cimentation de l'os et de l'implant est à privilégier, la méthode d'application et de pressurisation, manuelle ou pressurisée, restant au choix du chirurgien. En résumé, il semblerait que nos pratiques, bien qu'hétérogènes, soient acceptables excepté une trop faible utilisation du lavage pulsé. 
Ce travail présente certaines limites. Les résultats sont issus d'une enquête déclarative et peuvent donc ne pas être totalement représentatifs des pratiques. Toutefois, la véracité de certaines données (volume d'activité, type de ciment utilisé, préparation du ciment) a été confirmée par les extractions des données de traçabilité (ciment et implants) et les dispositifs référencés dans notre établissement (pistolets, système de mélange sous vide). Egalement, bien que réalisée sur plusieurs sites d'un $\mathrm{CHU}$, cette enquête reste monocentrique, la disponibilité des ciments et matériels associés (pistolets, systèmes de mélange, etc.) étant similaire sur les cinq sites. Il serait intéressant d'élargir cette enquête pilote à l'échelon régional voire national. Le taux de réponse était cependant satisfaisant et comparable à des enquêtes étrangères ayant également porté sur l'utilisation des ciments orthopédiques [79,23]. De plus, les chirurgiens n'ayant pas répondu à notre enquête étaient en grande partie des assistants, dont l'expérience est limitée et le volume d'activité plus faible que des praticiens hospitaliers.

\section{Conclusion}

Ce travail nous a permis de montrer une grande hétérogénéité des techniques de cimentation dans un $\mathrm{CHU}$ français, hétérogénéité également rapportée dans des enquêtes étrangères. Ceci est la conséquence du manque de recommandations claires de la part des sociétés savantes, et de données variées, parfois contradictoires dans la littérature. Cependant, un partage d'expérience en vue d'une harmonisation des techniques serait souhaitable. Aussi, une enquête multicentrique à l'échelle nationale serait pertinente pour évaluer les pratiques françaises.

Conflits d'intérêts : JL Besse : consultant pour Wright-Tornier. MH Fessy : consultant pour Serf, Depuy. Anthony Viste a un soutien financier sans bénéfice direct de Adler. Emmanuelle Carre a des soutiens financiers sans bénéfice direct de Adler et Biomet. Marine Barral a un soutien financier sans bénéfice direct de Newbone. Les autres auteurs n'ont rien à déclarer en relation ou en dehors de ce travail.

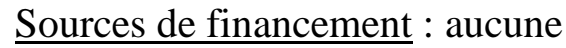

Contribution des auteurs : EC et AV étaient à l'initiative de ce projet. $\mathrm{MB}$ et $\mathrm{EC}$ ont conçu le questionnaire, validé par AV. MB et EC ont collecté les données. Elles ont été analysées 
par MB, LL, EC, AV et JLB. La préparation du manuscrit a été faite par MB, EC, LL, AV, JLB et MHF. Tous les auteurs ont lu et validé la version finale et soumise du manuscrit. 


\section{Références}

1. Schlegel UJ, Bishop NE, Püschel K, Morlock MM, Nagel K. Comparison of different cement application techniques for tibial component fixation in TKA. Int Orthop 2015;39:47-54.

2. Vaninbroukx M, Labey L, Innocenti B, Bellemans J. Cementing the femoral component in total knee arthroplasty: which technique is the best? The Knee 2009;16:265-8.

3. Delaunay C, Hamadouche M, Girard J, Duhamel A, SoFCOT Group. What are the causes for failures of primary hip arthroplasties in France? Clin Orthop Relat Res 2013;471:3863-9.

4. Argenson JN, Boisgard S, Parratte S, Descamps S, Bercovy M, Bonnevialle P, et al. Survival analysis of total knee arthroplasty at a minimum 10 years' follow-up: a multicenter French nationwide study including 846 cases. Orthop Traumatol Surg Res 2013;99:385-90.

5. Lohmann $\mathrm{CH}$, Hameister R, Singh G. Allergies in orthopaedic and trauma surgery. Orthop Traumatol Surg Res 2017;103(1, Supplement):S75-81.

6. Russotti GM, Coventry MB, Stauffer RN. Cemented total hip arthroplasty with contemporary techniques. A five-year minimum follow-up study. Clin Orthop Relat Res 1988;235:141-7.

7. Phillips AM, Goddard NJ, Tomlinson JE. Current techniques in total knee replacement: results of a national survey. Ann R Coll Surg Engl 1996;78:515-20.

8. Nedungayil SK, Mehendele S, Gheduzzi S, Learmonth ID. Femoral cementing techniques: current trends in the UK. Ann R Coll Surg Engl 2006;88:127-30.

9. Fischer CA, Kaszap B, Drexler C, Lehner B, Clarius M. Cemented total hip arthroplasty in Germany - update 2010. Z Orthopadie Unfallchirurgie 2012;150:309-17. 
10. Haute Autorité de Santé. Evaluation des ciments avec ou sans antibiotiques pour la fixation des implants articulaires [Internet]. Saint-Denis La Plaine : HAS ; 2016. Available from: https://www.has-sante.fr/portail/upload/docs/application/pdf/2017-02/rapport_ciment_vf.pdf

11. Gergely RCR, Toohey KS, Jones ME, Small SR, Berend ME. Towards the optimization of the preparation procedures of PMMA bone cement. J Orthop Res 2016;34:915-23.

12. Malchau H, Herberts $P$, Ahnfelt L. Prognosis of total hip replacement in Sweden. Follow-up of 92,675 operations performed 1978-1990. Acta Orthop Scand 1993;64:497-506.

13. Ungers LJ, Vendrely TG, Barnes CL. Control of methyl methacrylate during the preparation of orthopedic bone cements. J Occup Environ Hyg 2007;4:272-80.

14. Schlegel UJ, Siewe J, Delank KS, Eysel P, Püschel K, Morlock MM, de Uhlenbrock AG. Pulsed lavage improves fixation strength of cemented tibial components. Int Orthop 2011;35:1165-9.

15. Breusch SJ, Norman TL, Schneider U, Reitzel T, Blaha JD, Lukoschek M. Lavage technique in total hip arthroplasty: jet lavage produces better cement penetration than syringe lavage in the proximal femur. J Arthroplasty 2000;15:921-7.

16. Niki Y, Matsumoto H, Otani T, Tomatsu T, Toyama Y. How much sterile saline should be used for efficient lavage during total knee arthroplasty? Effects of pulse lavage irrigation on removal of bone and cement debris. J Arthroplasty 2007;22:95-9.

17. Walker PS, Soudry M, Ewald FC, McVickar H. Control of cement penetration in total knee arthroplasty. Clin Orthop Relat Res 1984;185:155-64.

18. Vanlommel J, Luyckx JP, Labey L, Innocenti B, De Corte R, Bellemans J. Cementing the tibial component in total knee arthroplasty: which technique is the best? J Arthroplasty 2011;26:492-6. 
19. Lutz MJ, Pincus PF, Whitehouse SL, Halliday BR. The effect of cement gun and cement syringe use on the tibial cement mantle in total knee arthroplasty. J Arthroplasty 2009;24:461-7.

20. Mann KA, Miller MA, Pray CL, Verdonschot N, Janssen D. A new approach to quantify trabecular resorption adjacent to cemented knee arthroplasty. J Biomech 2012;45:711-5.

21. Flivik G, Wulff K, Sanfridsson J, Ryd L. Improved acetabular pressurization gives better cement penetration: in vivo measurements during total hip arthroplasty. J Arthroplasty 2004;19:911-8.

22. Oh I, Merckx DB, Harris WH. Acetabular cement compactor. An experimental study of pressurization of cement in the acetabulum in total hip arthroplasty. Clin Orthop Relat Res 1983;177:289-93.

23. Lutz MJ, Halliday BR. Survey of current cementing techniques in total knee replacement. ANZ J Surg 2002;72:437-9. 
Tableau 1 Caractéristiques des répondeurs

\begin{tabular}{lc}
\hline Nombre de répondeurs, $\mathrm{n}$ & 21 \\
\hline Homme, $\mathrm{n}$ & 20 \\
\hline Age, moyenne \pm SD (min-max) & $41 \pm 10$ (31-59) \\
\hline Qualité du praticien & 5 \\
$\quad$ Professeur, $\mathrm{n}$ & 10 \\
$\quad$ Praticien hospitalier, $\mathrm{n}$ & 6 \\
$\quad$ Chef de Clinique Assistant, $\mathrm{n}$ & \\
\hline Années d'expérience, moyenne $\pm \mathrm{SD}$ (min- & $11 \pm 10(1-29)$ \\
max) & \\
\hline SD $=$ standard deviation
\end{tabular}


Tableau 2 Volume d'activité par type d'implant en 2017

\begin{tabular}{lcccc}
\hline & & Nombre de poses/an & Nombre de poses & Taux de poses \\
Type d'implant & $\begin{array}{c}\text { Nombre de } \\
\text { chirurgiens }\end{array}$ & $\begin{array}{c}\text { par chirurgien, } \\
\text { médiane (min; max })\end{array}$ & $\begin{array}{c}\text { chirurgien, médiane } \\
\text { (min; max) }\end{array}$ & $\begin{array}{c}\text { cimentées, médiane } \\
\text { (min; max) }\end{array}$ \\
\hline PTH primaire & $\mathrm{N}=19$ & $45(1-429)$ & $0(0-21)$ & $0 \%(0-14)$ \\
PTH reprise & $\mathrm{N}=19$ & $9(1-112)$ & $1(0-10)$ & $10 \%(0-100)$ \\
\hline PTG primaire & $\mathrm{N}=18$ & $78(5-218)$ & $55(0-218)$ & $92 \%(0-100)$ \\
PTG reprise & $\mathrm{N}=16$ & $8(2-40)$ & $8(1-40)$ & $100 \%(50-100)$ \\
\hline PTE primaire & $\mathrm{N}=5$ & $7(1-18)$ & $2(0-3)$ & $13 \%(0-100)$ \\
PTE reprise & $\mathrm{N}=2$ & $1(0-1)$ & $1(0-1)$ & $50 \%(0-100)$ \\
\hline PTCo primaire & $\mathrm{N}=1$ & 3 & $3(3-3)$ & $3 \%(0-7)$ \\
\hline PTCh primaire & $\mathrm{N}=2$ & $27(9-44)$ & $2(0-3)$ & $100 \%$
\end{tabular}

PTCh, prothèse totale de cheville; PTCo, prothèse totale de coude; PTE, prothèse totale d'épaule; PTG, prothèse totale de genou; PTH, prothèse totale de hanche 
Tableau 3 Ciments utilisés et techniques de cimentation par chirurgien

\begin{tabular}{|c|c|c|c|c|c|c|c|c|c|c|c|c|c|}
\hline Site & $\begin{array}{c}\text { Qualité } \\
\text { du } \\
\text { praticien }\end{array}$ & $\begin{array}{l}\text { Expé- } \\
\text { rience } \\
\text { (an) }\end{array}$ & Nom du ciment & $\begin{array}{c}\text { Nombre } \\
\text { implants } \\
\text { cimentés/ Total } \\
\text { PTH-I }{ }^{\text {aire }} \text {, } \\
\text { n/N (\%) }\end{array}$ & $\begin{array}{c}\text { Nombre } \\
\text { implants } \\
\text { cimentés/ Total } \\
\text { PTH-R, } \\
\text { n/N }(\%)\end{array}$ & $\begin{array}{c}\text { Nombre } \\
\text { implants } \\
\text { cimentés/ Total } \\
\text { PTG-I }{ }^{\text {aire }} \text {, } \\
\text { n/N (\%) }\end{array}$ & $\begin{array}{c}\text { Nombre } \\
\text { implants } \\
\text { cimentés/ Total } \\
\text { PTG-R, } \\
\text { n/N }(\%)\end{array}$ & $\begin{array}{c}\text { Nombre } \\
\text { implants } \\
\text { cimentés/ Total } \\
\text { Autre (PTE, } \\
\text { PTCo, PTCh), } \\
\text { n/N }(\%)\end{array}$ & $\begin{array}{l}\text { Lavage } \\
\text { pulsé }\end{array}$ & $\begin{array}{c}\text { Méthode } \\
\text { d'application }\end{array}$ & $\begin{array}{c}\text { Zone } \\
\text { d'application }\end{array}$ & $\begin{array}{l}\text { Méthode de } \\
\text { pressurisation }\end{array}$ & $\begin{array}{c}\text { Cimentation } \\
\text { des quilles } \\
\text { tibiales }\end{array}$ \\
\hline 1 & PU-PH ${ }^{\S}$ & 27 & Palacos $R+G$ & $21 / 429(5 \%)$ & $13 / 115(11 \%)$ & 0 & 0 & 0 & non & Pistolet (PTH) & Os et implant & Compresse & NA \\
\hline 1 & $\mathrm{PH}$ & 7 & Palacos $R+G$ & $0 / 61(0 \%)$ & $0 / 6(0 \%)$ & $70 / 105(67 \%)$ & $12 / 12(100 \%)$ & 0 & non & $\begin{array}{c}\text { Pistolet }(\mathrm{PTH}), \\
\text { spatule (PTG) }\end{array}$ & Os et implant & Spatule & En partie \\
\hline \multirow[t]{2}{*}{1} & \multirow{2}{*}{$\mathrm{CCA}$} & \multirow[t]{2}{*}{2} & Palacos $R+G$ & $2 / 63(3 \%)$ & $3 / 17(18 \%)$ & $6 / 7(86 \%)$ & \multirow{2}{*}{$3 / 3(100 \%)$} & $\begin{array}{c}\text { PTE-I }^{\text {aire }}, 1 / 1 \\
(100 \%)\end{array}$ & non & Manuel & Os et implant & $\begin{array}{l}\text { Manuel ou } \\
\text { compresse }\end{array}$ & En partie \\
\hline & & & Refobacin LV* & 0 & 0 & 0 & & 0 & NA & Manuel & NA & NA & NA \\
\hline 1 & PHU & 6 & Palacos $R+G$ & $0 / 15(0 \%)$ & $0 / 6(0 \%)$ & $3 / 25(12 \%)$ & $7 / 7(100 \%)$ & 0 & oui & Manuel & Os et implant & $\begin{array}{l}\text { Manuel ou } \\
\text { spatule }\end{array}$ & En totalité \\
\hline 1 & CCA & 1 & Palacos $R+G$ & $2 / 45(4 \%)$ & $0 / 10(0 \%)$ & $4 / 7(57 \%)$ & $3 / 3(100 \%)$ & $\begin{array}{c}\text { PTE-I }{ }^{\text {aire }}, 3 / 18 \\
(17 \%) \\
\text { PTE-R, } 1 / 1 \\
(100 \%)\end{array}$ & non & $\begin{array}{l}\text { Pistolet }(\mathrm{PTH}) \text { ou } \\
\text { manuel }\end{array}$ & $\begin{array}{l}\text { Os (PTE) sinon } \\
\text { os et implant }\end{array}$ & Compresse & En partie \\
\hline 1 & $\mathrm{PH}$ & 27 & Palacos $R+G$ & 0 & 0 & 0 & 0 & $\begin{array}{c}\text { PTCh, 3/44 } \\
(3 \%)\end{array}$ & non & Manuel & Os et implant & $\begin{array}{l}\text { Manuel ou } \\
\text { seringue }\end{array}$ & NA \\
\hline \multirow{2}{*}{1} & \multirow{2}{*}{$\mathrm{CCA}^{\S}$} & \multirow{2}{*}{2} & Palacos $R+G$ & $0 / 15(0 \%)$ & $0 / 9(0 \%)$ & $3 / 13(23 \%)$ & \multirow{2}{*}{$8 / 8(100 \%)$} & \multirow{2}{*}{0} & non & Manuel & Os et implant & Spatule & En totalité \\
\hline & & & Refobacin LV* & 0 & 0 & 0 & & & NA & Manuel & NA & NA & NA \\
\hline 2 & PU-PH ${ }^{\S}$ & 13 & Palacos $R+G$ & $0 / 2(0 \%)$ & $0 / 1(0 \%)$ & $84 / 86(98 \%)$ & 9/9 (100\%) & $\begin{array}{c}\text { PTE-I }^{\text {aire }}, 3 / 7 \\
(43 \%)\end{array}$ & oui & $\begin{array}{l}\text { Manuel et/ou } \\
\text { pistolet }\end{array}$ & Os et implant & Spatule & En partie \\
\hline 2 & PU-PH ${ }^{\S}$ & 11 & Palacos $R+G$ & $6 / 195(3 \%)$ & $1 / 10(10 \%)$ & $218 / 218(100 \%)$ & $11 / 11(100 \%)$ & 0 & oui & $\begin{array}{l}\text { Manuel et/ou } \\
\text { pistolet }\end{array}$ & Os et implant & Spatule & En partie \\
\hline 2 & $\mathrm{CCA}$ & 1 & Palacos $R+G$ & $0 / 40(0 \%)$ & $10 / 20(50 \%)$ & $60 / 60(100 \%)$ & $40 / 40(100 \%)$ & 0 & oui & $\begin{array}{l}\text { Manuel et/ou } \\
\text { pistolet }\end{array}$ & Os et implant & Spatule & En partie \\
\hline 2 & $\mathrm{CCA}^{\S}$ & 2 & Palacos $R+G$ & $3 / 24(13 \%)$ & $0 / 1(0 \%)$ & 92/94 (98\%) & $16 / 16(100 \%)$ & 0 & oui & $\begin{array}{c}\text { Manuel et/ou } \\
\text { pistolet }\end{array}$ & Os et implant & Spatule & En partie \\
\hline 2 & $\mathrm{CCA}^{\S}$ & 2 & Palacos $R+G$ & $2 / 23(9 \%)$ & $0 / 9(0 \%)$ & $85 / 85(100 \%)$ & $7 / 7(100 \%)$ & 0 & oui & $\begin{array}{l}\text { Manuel et/ou } \\
\text { pistolet }\end{array}$ & Os et implant & Spatule & En partie \\
\hline 3 & $\mathrm{PHU}^{\S}$ & 7 & Palacos $R+G$ & $9 / 63(14 \%)$ & $6 / 7(86 \%)$ & $14 / 48(29 \%)$ & $7 / 14(50 \%)$ & 0 & oui & $\begin{array}{l}\text { Manuel et/ou } \\
\text { pistolet }\end{array}$ & Os et implant & NR & En totalité \\
\hline
\end{tabular}




\begin{tabular}{|c|c|c|c|c|c|c|c|c|c|c|c|c|c|}
\hline Site & $\begin{array}{c}\text { Qualité } \\
\text { du } \\
\text { praticien }\end{array}$ & $\begin{array}{l}\text { Expé- } \\
\text { rience } \\
\text { (an) }\end{array}$ & Nom du ciment & $\begin{array}{l}\text { Nombre } \\
\text { implants } \\
\text { cimentés/ Total } \\
\text { PTH-I } \\
\text { n/N }(\%)\end{array}$ & $\begin{array}{c}\text { Nombre } \\
\text { implants } \\
\text { cimentés/ Total } \\
\text { PTH-R, } \\
\text { n/N }(\%)\end{array}$ & $\begin{array}{c}\text { Nombre } \\
\text { implants } \\
\text { cimentés/ Total } \\
\text { PTG-I }{ }^{\text {aire }} \text {, } \\
\text { n/N (\%) }\end{array}$ & $\begin{array}{c}\text { Nombre } \\
\text { implants } \\
\text { cimentés/ Total } \\
\text { PTG-R, } \\
\text { n/N }(\%)\end{array}$ & $\begin{array}{c}\text { Nombre } \\
\text { implants } \\
\text { cimentés/ Total } \\
\text { Autre (PTE, } \\
\text { PTCo, PTCh), } \\
\text { n/N (\%) }\end{array}$ & $\begin{array}{c}\text { Lavage } \\
\text { pulsé }\end{array}$ & $\begin{array}{c}\text { Méthode } \\
\text { d'application }\end{array}$ & $\begin{array}{c}\text { Zone } \\
\text { d'application }\end{array}$ & $\begin{array}{l}\text { Méthode de } \\
\text { pressurisation }\end{array}$ & $\begin{array}{c}\text { Cimentation } \\
\text { des quilles } \\
\text { tibiales }\end{array}$ \\
\hline 3 & PU-PH ${ }^{\S}$ & 29 & Simplex & 0 & 0 & 0 & 0 & $\begin{array}{c}\frac{\text { PTE- I }}{\text { aire }, 0 / 10} \\
\underline{(0 \%)} \\
\frac{\text { PTE-R, } 0 / 1}{(0 \%)} \\
\frac{\text { PTCo, } 3 / 3}{(100 \%)}\end{array}$ & non & NR & Os & Autre & NA \\
\hline 3 & $\mathrm{PH}$ & 5 & Palacos $R+G$ & $0 / 40(0 \%)$ & $1 / 4(25 \%)$ & $0 / 20(0 \%)$ & $1 / 2(50 \%)$ & 0 & non & Manuel & Implant & NR & En partie \\
\hline 4 & PU-PH & 16 & $\begin{array}{c}\text { Palacos } R+G \\
\text { Palacos } L V+G\end{array}$ & $0 / 1(0 \%)$ & $2 / 2(100 \%)$ & $5 / 5(100 \%)$ & 0 & $\begin{array}{c}\text { PTE- I }{ }^{\text {aire }}, 2 / 2 \\
(100 \%)\end{array}$ & non & Pistolet & Os & NR & En partie \\
\hline 5 & $\mathrm{PH}$ & 11 & Refobacin R Genta & $0 / 137(0 \%)$ & $4 / 11(36 \%)$ & $168 / 168(100 \%)$ & $6 / 6(100 \%)$ & 0 & oui & $\begin{array}{c}\text { Manuel, } \\
\text { ostéotome ou } \\
\text { pistolet (PTH) }\end{array}$ & Os et implant & $\begin{array}{c}\text { Manuel ou } \\
\text { pistolet (PTH) }\end{array}$ & En totalité \\
\hline 5 & $\mathrm{PH}^{\S}$ & 7 & Refobacin R Genta & $1 / 136(4 \%)$ & $0 / 7(0 \%)$ & $128 / 128(100 \%)$ & $8 / 8(100 \%)$ & 0 & oui & $\begin{array}{c}\text { Manuel, } \\
\text { ostéotome ou } \\
\text { pistolet (PTH) }\end{array}$ & Os et implant & $\begin{array}{c}\text { Manuel ou } \\
\text { pistolet (PTH) }\end{array}$ & En totalité \\
\hline 5 & $\mathrm{PH}$ & 27 & Refobacin R Genta & $0 / 152(0 \%)$ & $1 / 10(10 \%)$ & $203 / 203(100 \%)$ & $15 / 15(100 \%)$ & 0 & oui & $\begin{array}{c}\text { Manuel, } \\
\text { ostéotome ou } \\
\text { pistolet (PTH) }\end{array}$ & Os et implant & $\begin{array}{c}\text { Manuel ou } \\
\text { pistolet (PTH) }\end{array}$ & En totalité \\
\hline 5 & $\mathrm{PH}^{\S}$ & 11 & Refobacin R Genta & $0 / 38(0 \%)$ & $1 / 4(25 \%)$ & $50 / 71(70 \%)$ & 0 & PTCh, 0/9 (0\%) & oui & $\begin{array}{c}\text { Manuel, } \\
\text { ostéotome ou } \\
\text { pistolet (PTH) }\end{array}$ & Os et implant & $\begin{array}{c}\text { Manuel ou } \\
\text { pistolet (PTH) }\end{array}$ & En totalité \\
\hline 5 & $\mathrm{PH}^{\S}$ & 29 & Refobacin R Genta & $0 / 209(0 \%)$ & $3 / 18(17 \%)$ & $17 / 165(10 \%)$ & $2 / 2(100 \%)$ & 0 & oui & $\begin{array}{c}\text { Manuel, } \\
\text { ostéotome ou } \\
\text { pistolet (PTH) }\end{array}$ & Os et implant & $\begin{array}{c}\text { Manuel ou } \\
\text { pistolet (PTH) }\end{array}$ & En totalité \\
\hline
\end{tabular}

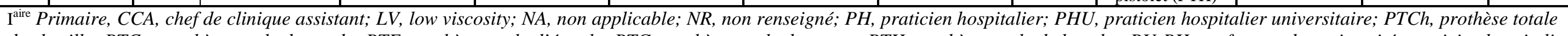

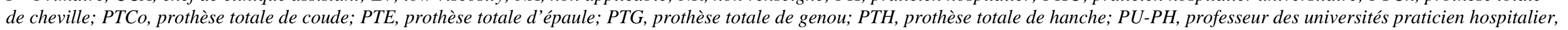
$-R$, Reprise.

*Refobacin LV utilisé comme espaceur dans les chirurgies en deux temps.

§Chirurgiens pour lesquels nous avons eu recours aux données de traçabilité pour évaluer leurs activités chirurgicales en 2017. 
Tableau 4. Comparatif des enquêtes par questionnaire publiées sur les ciments et les techniques de cimentation

\begin{tabular}{|c|c|c|c|c|c|}
\hline Auteurs & Phillips et al. [7] & Lutz et al. [23] & Nedungayil et al. [8] & Fischer et al. [9] & Notre étude \\
\hline Pays & Royaume-Uni & Australie & Royaume-Uni & Allemagne & France \\
\hline Niveau & National & Régional (Queensland) & National & National & Local \\
\hline $\begin{array}{l}\text { Date de } \\
\text { publication }\end{array}$ & 1996 & 2002 & 2006 & 2010 & 2018 \\
\hline $\begin{array}{l}\text { Nombre de } \\
\text { répondeurs, n } \\
(\%)\end{array}$ & $597 / 1162(62 \%)$ & $110 / 129(85 \%)$ & $762 / 1620(47 \%)$ & $255 / 492(52 \%)$ & $21 / 34(62 \%)$ \\
\hline Type d'implant & PTG (reprises) & PTG (reprises) & $\begin{array}{l}\text { PTH, dont } 77 \% \text { de } \\
\text { reprises }\end{array}$ & PTH & $\begin{array}{c}\text { PTH, PTG, PTE, PTCo et } \\
\text { PTCh (primaire } \\
\text { et reprise) }\end{array}$ \\
\hline $\begin{array}{l}\text { Fréquence } \\
\text { d'utilisation des } \\
\text { ciments }\end{array}$ & $\begin{array}{l}\text { Moyenne : } 34,3 / \text { an } \\
\text { La plupart des chirurgiens } \\
\text { en font entre }[20-40]\end{array}$ & NR & $\begin{array}{l}31 \% \text { des chirurgiens : } \\
\text { moins de } 30 \mathrm{PTH} / \mathrm{an} \\
27 \%: 30-50 / \mathrm{an} \\
15 \%: 50-70 / \mathrm{an}\end{array}$ & $\begin{array}{l}40 \% \text { des centres } \\
\text { cimentaient plus de } 40 \% \\
\text { des PTH chaque année. }\end{array}$ & 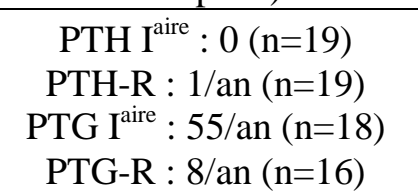 \\
\hline $\begin{array}{l}\text { Utilisateurs de } \\
\text { ciment parmi les } \\
\text { répondeurs }\end{array}$ & $567 / 597(95 \%)$ & $77 / 110(70 \%)$ & $568 / 762(75 \%)$ & NR & $21 / 21(100 \%)$ \\
\hline $\begin{array}{l}\text { Ciment utilisé } \\
\text { majoritairement }\end{array}$ & Palacos R+G (59\%) & NR & $\begin{array}{c}\text { Ciment haute viscosité } \\
(82 \%) \text {, avec antibiotique } \\
(77 \%) \\
\text { Palacos } \mathrm{R}+\mathrm{G}(59 \%)\end{array}$ & Palacos R+G (52\%) & $\begin{array}{l}\text { Ciment haute viscosité } \\
\text { avec gentamicine }(95 \%)\end{array}$ \\
\hline $\begin{array}{l}\text { Mélange ciment } \\
\text { avec un système } \\
\text { sous vide }\end{array}$ & NR & NR & Oui $(94 \%)$ & Oui $(85 \%)$ & Non \\
\hline Lavage pulsé & $244(43 \%)$ & $52(68 \%)$ & Fémur : 494 (87\%) & $\begin{array}{c}\text { Fémur : } 199(78 \%) \\
\text { Acétabulum : } 170(67 \%)\end{array}$ & $12(57 \%)$ \\
\hline Séchage de l'os & $499(88 \%)$ & $71(92 \%)$ & NR & NR & NR \\
\hline
\end{tabular}




\begin{tabular}{|c|c|c|c|c|c|}
\hline Auteurs & Phillips et al. [7] & Lutz et al. [23] & Nedungayil et al. [8] & Fischer et al. [9] & Notre étude \\
\hline $\begin{array}{l}\text { Double } \\
\text { cimentation } \\
\text { (os+implant) }\end{array}$ & $\begin{array}{l}\text { Côté fémoral : } 62 \% \\
\text { Côté tibial : } 33 \% \\
\text { aucun consensus }\end{array}$ & NR & NR & NR & $86 \%$ \\
\hline Implant cimenté & $\begin{array}{l}95 \% \text { pour patella et tibia } \\
80 \% \text { fémur }\end{array}$ & Tibia & Fémur & Acétabulum et fémur & NR \\
\hline Pressurisation & NA & $\begin{array}{l}\text { Tibial : pistolet (5\%), } \\
\text { autres }\end{array}$ & $\begin{array}{l}\text { Variée : manuelle }(33 \%), \\
\text { dispositif pressurisation } \\
(30 \%) \text {, les deux }(36 \%)\end{array}$ & $\begin{array}{l}\text { Acétabulum : manuelle } \\
\qquad(89 \%)\end{array}$ & $\begin{array}{l}\text { Manuelle ou pistolet } \\
\text { (PTH) }\end{array}$ \\
\hline
\end{tabular}

$\mathrm{I}^{\mathrm{aire}}$ Primaire; NR, non renseigné; PTCh, prothèse totale de cheville; PTCo, prothèse totale de coude; PTE, prothèse totale d'épaule; PTG, prothèse totale de genou; PTH, prothèse totale de hanche, -R, Reprise. 


\section{Liste des figures}

Figure 1. Flowchart de la population étudiée

Légende: $C C A$, chef de clinique assistant; $P H$, praticien hospitalier; $P U-P H$, professeur des universitéspraticien hospitalier 


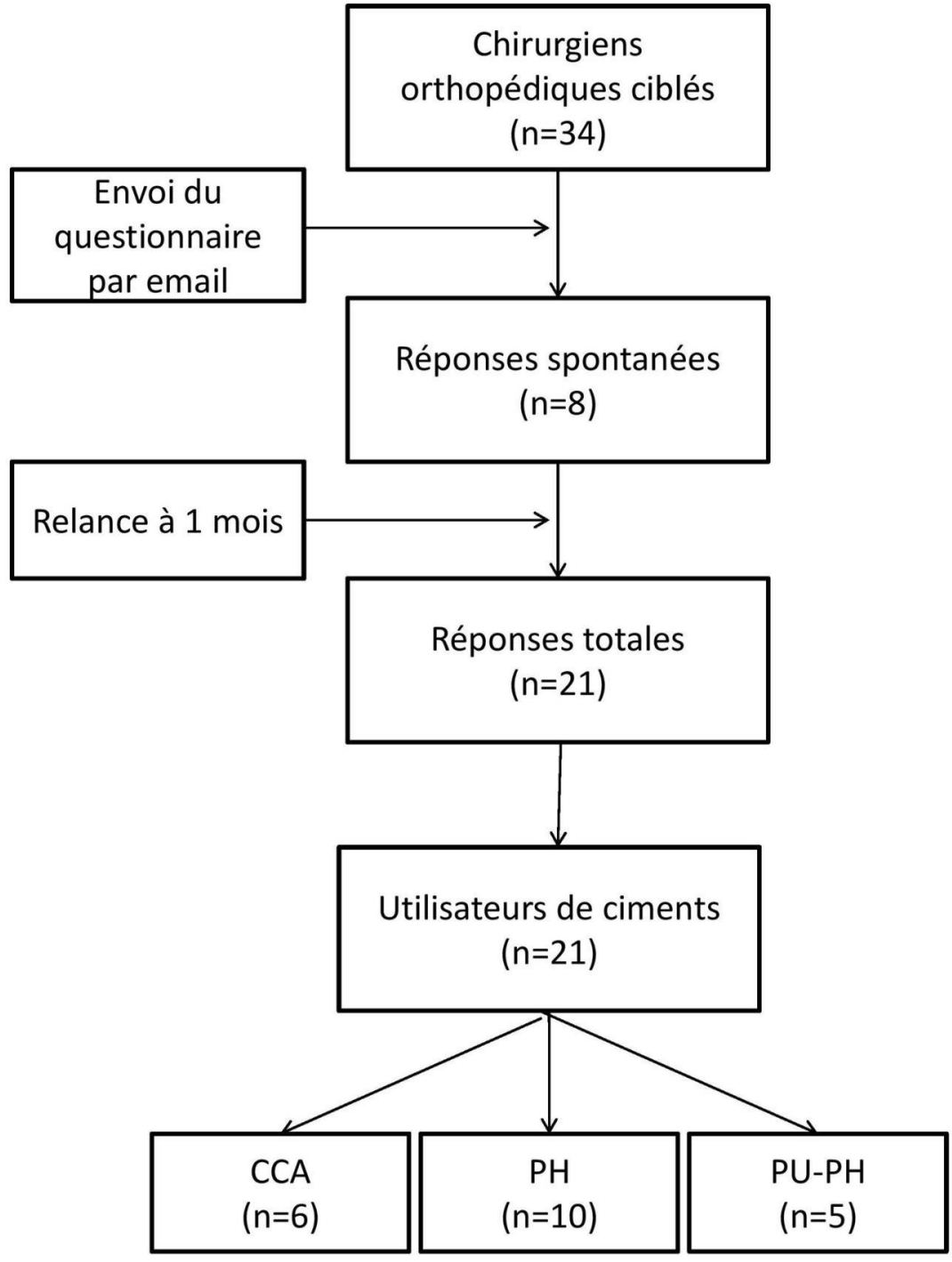

